\title{
Comparison of Alternative Representational Formats for Hyperlinks: Pictogram, Labeled-Pictogram, and Text
}

\author{
Miki Namatame ${ }^{*}, 1$ and Muneo Kitajima ${ }^{2}$ \\ ${ }^{I}$ Tsukuba University of Technology, 4-3-15 Amakubo Tsukuba Ibaraki 305-8520 Japan \\ ${ }^{2}$ National Institute of Advanced Industrial Science and Technology (AIST), 1-1-1 Higashi Tsukuba Ibaraki 305-8566 \\ Japan
}

\begin{abstract}
This paper reports an eye-tracking experiment conducted to compare alternative representations of directories typically shown on web pages in search of a best representation for deaf persons. The experiment simulated a directorybased information search task to understand how it is performed when directories are represented in text, labeledpictograms, or unlabeled-pictograms. Twenty-one deaf and 21 hearing participants were asked to select one of 27 directories represented in one of the three alternative formats for each of 38 queries. The eye movement data were analyzed by the parametric ANOVA to understand how the method of directory search adopted by the hearing group and the deaf group might be different under the influence of the differences in directory representations. The result demonstrated that only in the labeled-pictogram representation, the hearing group and the deaf group performed equally well in terms of the eye movement measures.
\end{abstract}

\section{INTRODUCTION}

The pervasiveness of the Web has been growing in line with continued advances in information technology, and an ever-growing amount of information has accumulated on the World Wide Web. At the same time, the need to make the information accessible to any person who needs it has become a serious issue. In the Web accessibility field, assuring accessibility not only for people with disabilities but also for elderly persons has become an important concern [1].

Directory-based information search is a widely used method to access information buried in an information structure. Fig. (1) shows an example of typical top pages of Web sites. This is from Yahoo! where a list of navigation links is provided on the left part of the page. These links serve as entrances to respective topics that a visitor wants to get information on those pages. In this example, the links are represented by pairs of a pictogram and a label that would best represent the topics that are reachable by starting navigation from these links. However, there has little been studied how effective this style of representation of navigation links is. The links can alternatively be represented by a list of text labels or a list of pictograms. It is known that there is a class of persons who are good at processing images, and there is another class of persons who are good at processing textual representations. These classes are not necessarily exclusive but it is important to notice that there are two classes which have different cognitive skills for processing a list of navigation links. A question arises. Which representation does best match which class? The effect of the different representations on the different classes

*Address correspondence to this author at the Tsukuba University of Technology, 4-3-15 Amakubo Tsukuba Ibaraki 305-8520 Japan;

E-mail:miki@a.tsukuba-tech.ac.jp might be negligible or large enough to affect the navigability of the sites. However, this issue might have great impact on accessibility issue for those who must be considered from the viewpoint of accessibility because this issue may emerge due to asymmetric cognitive skills of those persons. Some deaf --are good at processing images but very bad at processing text. To our knowledge, little investigation has been done on the alternative representation of navigation links from this perspective.

This paper tries to answer this question by comparing three alternative representations of hyper-links, i.e., text, labeled-pictograms, and unlabeled-pictograms, in terms of their utility for supporting directory-based information search tasks. A pictogram is a symbol representing a concept, object, activity, place or event by illustration. Examples of pictogram are shown in Fig. (2). Pictograms have been used at public spaces such as airports and train stations as a means for transmitting messages directly and instantly to passengers. Pictograms are intuitive visual representations of meanings and would have advantage over textual representations in those situations. We hypothesized that the advantage of pictograms may hold in directory selection tasks performed in web sites where quick navigability would be preferred.

This paper specifically focuses on the level of multimedia literacy of users concerning text and pictograms. The level of multimedia literacy might be defined in various ways. However, this paper exclusively focuses on text and images. Deaf persons use different cognitive processes than hearing persons when examining visual information [2], and they rely more heavily on visual representation than textual representation when examining the contents on web pages [3-7]. Therefore, this paper considers deaf persons as the representative users that use visual information more heavily than textual information and hearing persons as the deaf 


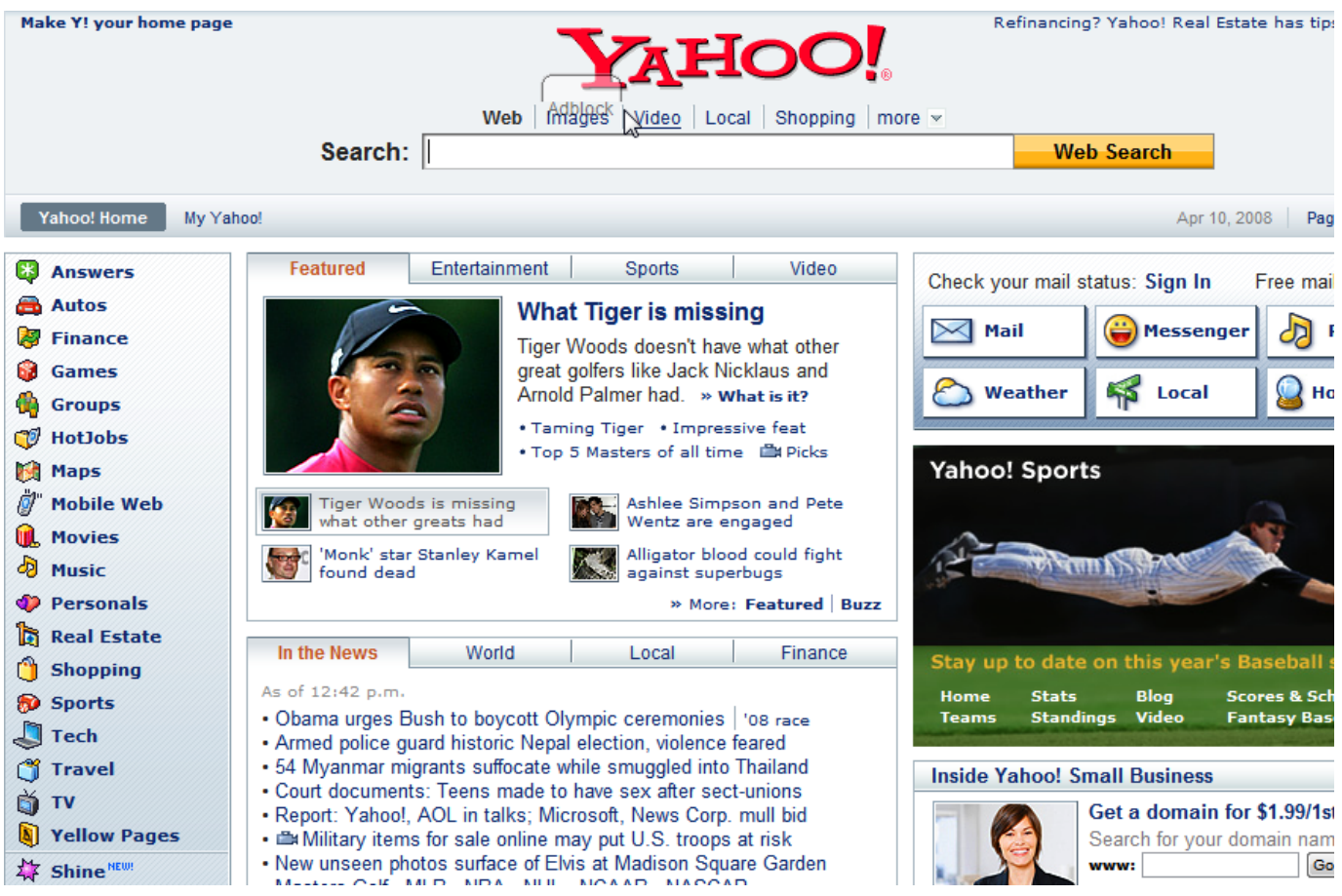

Fig. (1). A typical top page of a web site.

persons' counterparts along the multimedia literacy spectrum characterized by text and image usage [8].

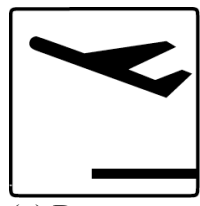

(a) Departures

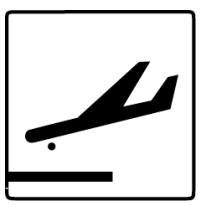

(b) Arrivals
Fig. (2). Examples of pictogram defined by JIS Z8210 (Japanese industrial standard).

This paper reports the result of an eye-tracking experiment that investigated the effect of alternative representations of directories on the users' performance of a directory-based information search task from the viewpoint of speed of directory selection, the variability of the selected directories for given queries, and the eye movement measures such as the number of eye fixations and the duration of eye fixations.

\section{EXPERIMENT}

\section{Participants}

Twenty-one deaf persons and 21 hearing persons participated in the experiment. The deaf participants were undergraduate students of the first author's university. The hearing participants were undergraduate students of a

\section{Table 1. Queries Used in the Experiment}

\begin{tabular}{|c|c|c|c|}
\hline 1. & Want to know tomorrow's weather & & Want to make friends by using chat \\
\hline 2. & Want to know typhoon information & 21. & Want to learn how to cook Thai curry \\
\hline 3. & Want to know the results of preliminary matches for soccer world cup & 22 . & Want to volunteer for something \\
\hline & soccer & 23. & Want to obtain driver's license \\
\hline 4. & Want to know the results of yesterday's professional baseball games & 24. & Want to study abroad in US \\
\hline 5 . & Want to know how to transfer to a train for the Shibuya station & 25 . & Want to open a shop at flea market \\
\hline 6. & Want to see next week's TV programs & 26. & Want to learn hip-hop \\
\hline 7. & Want to go to see fortune-teller for niceness with a friend & 27. & Want to learn more about the medicine the doctor prescribed \\
\hline 8. & Want to purchase a bag made of Louis Vuitton & 28. & Want to make friends with, foreigners \\
\hline 9. & Want to order flower arrangements for Mother's day over Internet & 29. & Want to play DVD with PC \\
\hline 10. & Want to French restraints & 30. & Want to join a tour accompanied by a sign language interpreter \\
\hline 11. & Want to purchase a used-computer & 31. & Want to obtain a hearing dog \\
\hline 12. & Want to know ZIP code by using postal address & 32 . & Want to know the meaning of "Mei-Kyou-Shi-Sui" \\
\hline 13. & Want to know the location of a shop by using its postal address & 33. & Want to know the schedule of an art exhibition \\
\hline 14. & Want to request information about recruitment & & Want to search for a part time home-working job \\
\hline 15. & Want to confirm whether Shinkansen is operating normally & 35 . & Want to play Japanese chess over Internet \\
\hline 16. & Want to purchase a ticket for a J-league soccer match & 36. & Want to know about the latest situation of the Iraq war \\
\hline 17. & Want to know the timetable of the express highway bus & 37. & Want to learn the Russian language \\
\hline $\begin{array}{l}18 . \\
19 .\end{array}$ & $\begin{array}{l}\text { Want to purchase discount airline flight ticket } \\
\text { Want to move to a more larger room }\end{array}$ & 38. & Want to take examination for qualification of word processing skill \\
\hline
\end{tabular}




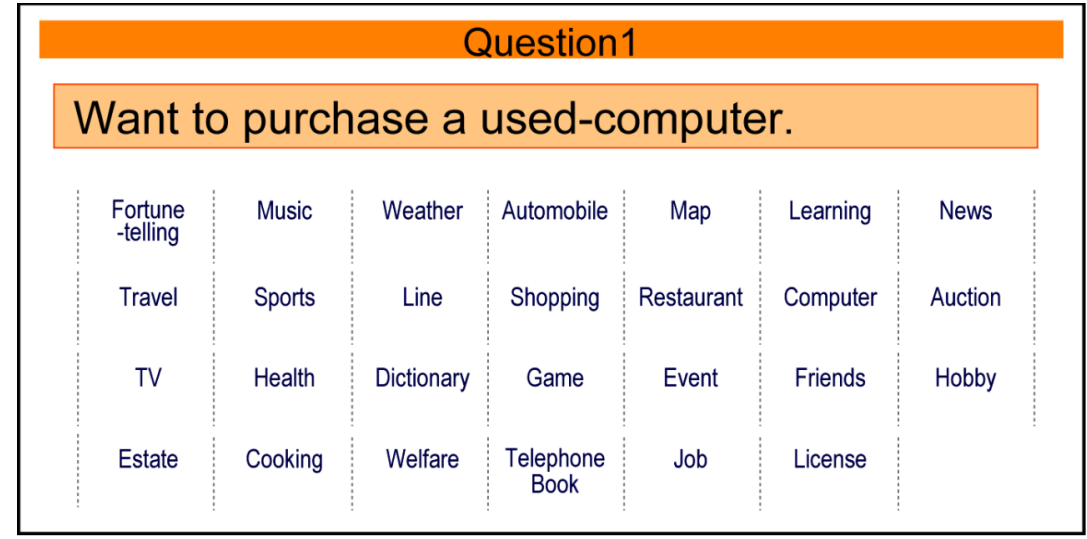

(a) Text

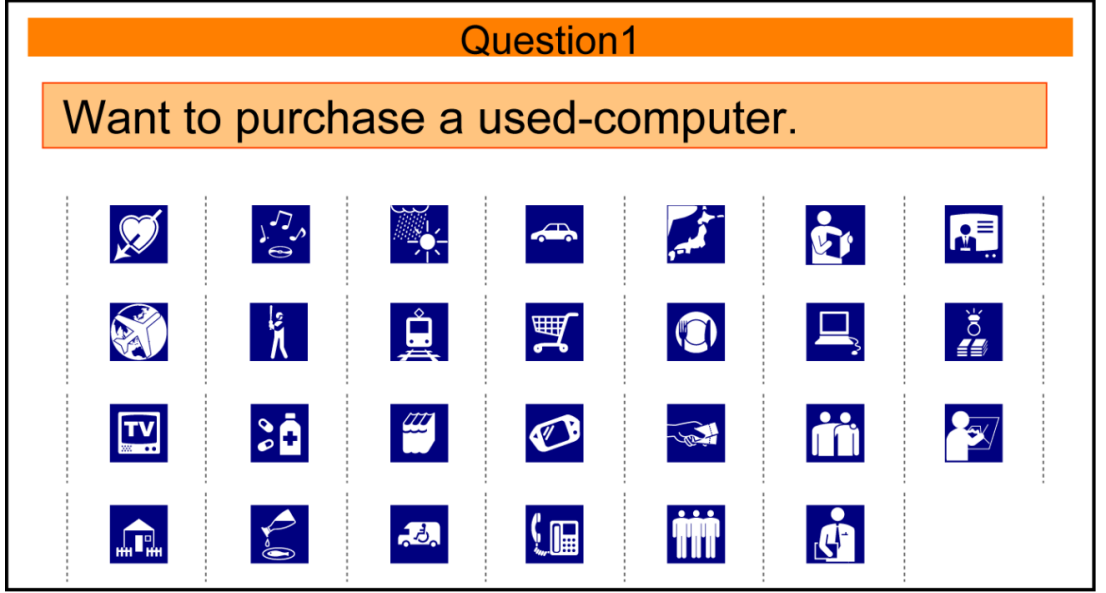

(b) Unlabeled Pictogram

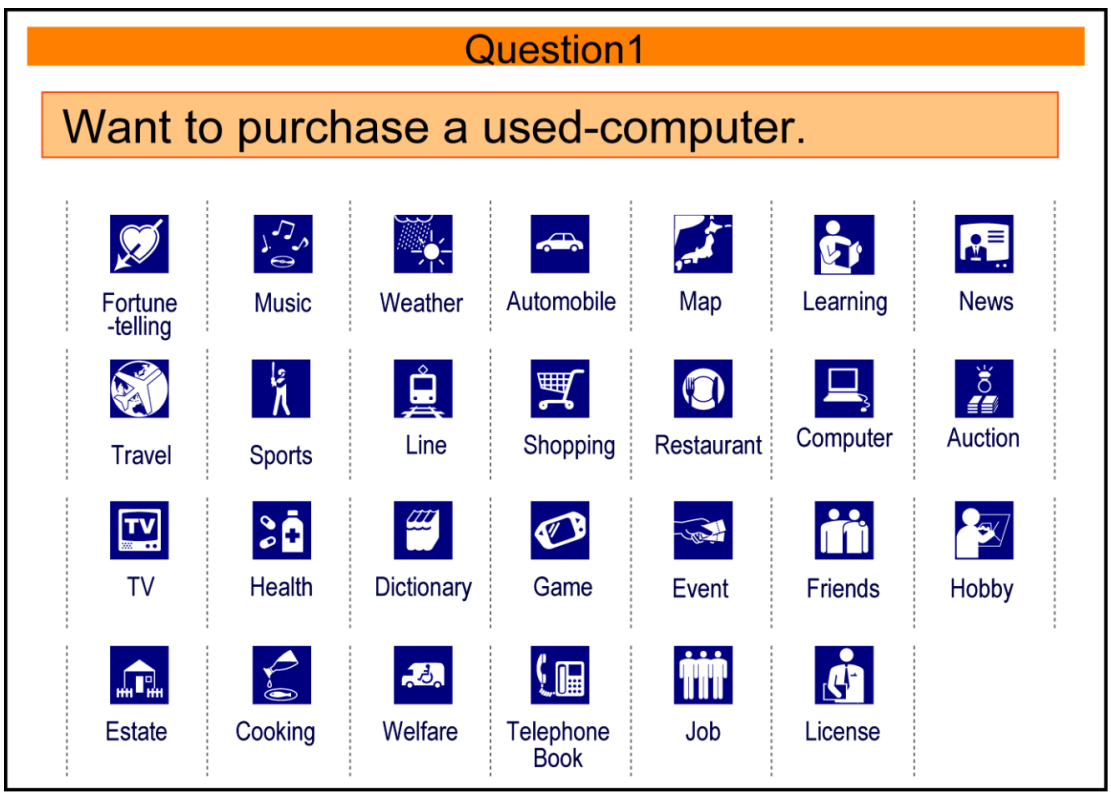

(c) Labeled Pictogram

Fig. (3). Alternative representations of directories.

university next to the first author's. All the participants were regular internet users and had an adequate performance level for using the Japanese language.

\section{Task and Stimuli}

The participants were asked to select a directory that best matched a query such as "Want to purchase a usedcomputer" from the 27 directories displayed on an 17 inch 
LCD monitor in one of three forms: 1) text (Fig. 3a) the unlabeled pictogram (Fig. 3b) labeled pictogram (Fig. 3c). The 27 directories were chosen from representative portal sites.

\section{Procedure}

Each participant was asked to select one of 27 directories for each of 38 queries (see Table 1). Task instruction was given through a page on the monitor. A practice session consisting of two queries was administered before starting a main session. The tasks were performed on a PC by clicking links on the pages. When clicking a link for a query, a page appeared with an indication to proceed by clicking the "Next Query" button. One third of the deaf participants and one third of the hearing participants were allocated to each of the three forms, text, unlabeled-pictogram, or labeled-pictogram.

\section{Equipment}

Participants' eye movements and mouse events were recorded with a Tobii 1750 eye tracker. The eye tracker didn't require the user to "do" or "wear" anything. All subjects could carry out the tasks without body-restriction. They simply sit in front of the eye tracker. Participant's gaze points on the screen were recorded at the rate of $50 \mathrm{~Hz}$.

\section{RESULTS}

The experimental data including eye movement data were analyzed by the parametric ANOVA to understand how the method of directory search adopted by the hearing group and the deaf group might be different under the influence of the differences in directory representations. We examined five performance measures to understand Web interaction characteristics of the deaf people or hearing people: 1) task completion time, 2) information theoretic entropy, 3) total length of fixations, 4) total number of fixations and 5) average fixation time.

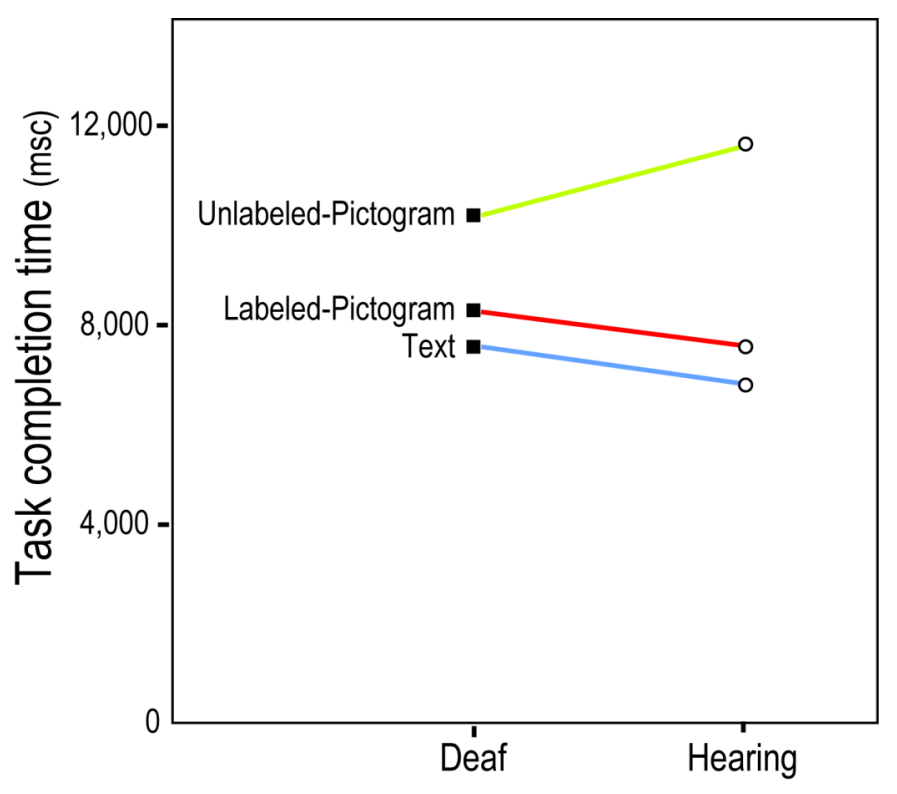

(a) Task completion time

\section{Task Completion Time}

The average time necessary for a participant to complete the task measures the speed of task performance. Fig. (4a) plots the average time necessary for completing a query in terms of characteristics of participants, deaf $v s$ hearing. Task completion time for the unlabeled-pictogram condition was significantly greater than the other two conditions. There were no significant differences between the task completion times of the hearing group and the had-of-hearing group. This result suggests that the unlabeled-pictogram representation should not be a good one for directory search in terms of speed of task performance.

\section{The Degree of Consistency of Selections}

The distribution of the frequencies of selected directories for a query that a group of participants selected using one of the three representations measures the degree of consistency with which the group understands the meaning of the query with reference to the specific representations of the directories in the respective formats. A group selecting a single, unique directory for a query understands the query consistently using the representation format. In contrast, a group selecting a variety of directories for a query perceives the meaning of the query less consistently, i.e., more ambiguously. We calculated the information theoretic entropy for measuring the degree of consistency. As the smaller the information theoretic entropy becomes, the more consistently the participant-group understands the query.

The procedure to derive entropy is as follows. Let $X_{r, g, k}(i)$ be the frequency of selecting the directory $i(1 \leq i \leq$ $27)$ for the query $k(1 \leq k \leq 38)$ in the representation form $r$ $\in$ \{unlabeled-pictogram, labeled-pictogram, text $\}$ by the participant-group $g \in$ \{deaf group, hearing group $\}$, then the probability of selecting directory $i$ for query $k$ by group $g$ in representation format $r$ is given by the following formula:

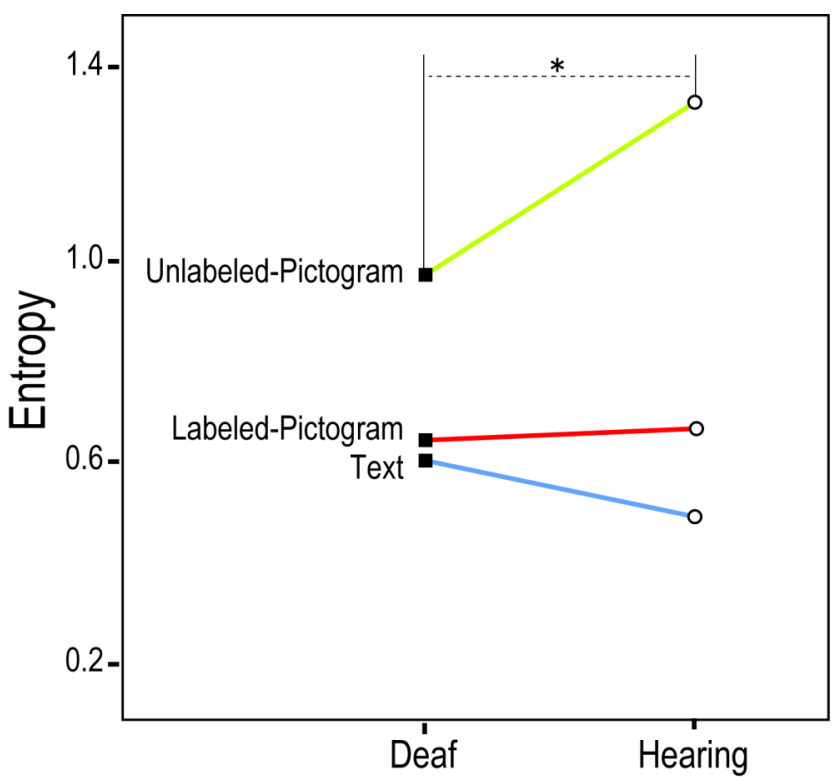

(b) Entropy

Fig. (4). Results of the experiment. 


$$
P_{r, g, k}(i)=\frac{X_{r, g, k}(i)}{\sum_{i=j}^{27} X_{r, g, k}(j)}
$$

By using this probability, information theoretic entropy of query $k$ for participant-group $g$ in representation form $r$, $H_{r, g, k}$, is given as follows:

$$
\begin{aligned}
& H_{r, g, k}=-\sum_{i=1}^{27} P_{r, g, k}(i) \log _{2} P_{r, g, k}(i), \text { where } \\
& 0 \leq H_{r, g, k} \leq 4.75=\log _{2} 27 \\
& H_{r, g, k} \quad \text { becomes smaller as the participant-group's }
\end{aligned}
$$
responses become more consistent. It takes the minimum value, 0 , when all the participants of the group $g$ selected a common directory for the query $k$ in representation form $r$. On the other hand, when the responses to the query $k$ from the participant-group $g$ distributed evenly to all the directories, it takes the maximum value, 4.75. Finally, we can obtain the average information theoretic entropy for queries in each representation form by averaging over the corresponding values of information theoretic entropy.

We obtained the average information theoretic entropy. Fig. (4b) demonstrates that the average entropy for the unlabeled-pictogram condition was significantly greater than those for the other conditions. In addition, the average entropy value of the unlabeled pictogram condition for the hearing group was significantly greater than that for the deaf group. This result suggests that the directories that the participants selected for the queries were more diverse for the unlabeled-pictogram condition than the other conditions and the disadvantage of the unlabeled-pictogram representation.

\section{Eye Movement Measures}

\section{Eye-Gaze Pattern}

So far, disadvantage of unlabeled-pictogram has been suggested but the goodness of the other two conditions, labeled-pictogram and text, has not been investigated by the task completion time and the entropy measures. However, as is shown below, eye movement measures were able to disentangle this issue. Fig. (5) shows typical gaze plots of the deaf participants for the text representation (Fig. 5a) and for the unlabeled-pictogram (Fig. 5b) who searched for the directory for the task "want to purchase a used-computer." The target was "computer" located at the second column from the right on the second row. In the figure, each fixation is depicted by a circle. A series of gaze points obtained in every $20 \mathrm{msec}$ as $\mathrm{X}$ - Y- coordinates on the screen were judged as a fixation if they located close each other within a circle of the radius of 30 pixels and the number of successive gazes in that region was more than five, in other words, if the eyes stayed within the circle longer than $100 \mathrm{msec}$, a cluster of the gazes was judged as a fixation. The radius of the circle in the figure is proportional to the duration of the fixation. The numbers in the circles show the order of fixations. The fixations are connected as they appeared.
The gaze plot of Fig. (5a) is for the text representation, that of Fig. (5) is for the unlabeled-pictogram representation, and that of Fig. (5c) is for the labeledpictogram representation. There are clear differences among them. The gaze plot of the text representation, Fig. (5a), shows a simple scan path with 9 fixations. It seems that the participant selected the correct one after examining the other three candidates. On the other hand, the gaze plot of the unlabeled pictogram representations, Fig. (5b), shows 30 fixations. The scan path shows that the participant examined "approximately" each of the pictograms orderly, not strictly left-to-right but with some order. The gaze plot of the labeled-pictogram representation, Fig. (5c), shows the smoothest scan path in which the participant's eyes almost directly dropped on the target pictogram, moved to its label, and returned to the pictogram to click it. We speculate that the unlabeled-pictogram representation had heavier load on information search than the text representation and that the unlabeled-pictogram representation needed longer time to complete the task than the text representation. These speculations will be further examined in the following subsections.

\section{Total Length of Fixations}

Fig. (6a) demonstrates that the deaf group showed longer total fixations than the hearing group $(F(1,37)=11.33$, $p<.01)$, and there were significant differences in the representations $(F(2,74)=19.20, \quad p<.01)$. The labeledpictogram showed significantly shorter total fixations than the other two representations and the unlabeled-pictogram representation showed significantly longer total fixations than the other two representations. However, we find no correlation between the participant's group and the representations.

\section{Total Number of Fixations}

Fig. (6b) demonstrates that there were significant differences in the representations $(F(2,74)=17.76, p<.01)$. The unlabeled-pictogram representation resulted in more fixations than the text or the labeled-pictogram representations. However, there was no significant difference between the groups. The fixation points of text representations were almost the same between the groups.

\section{Average Fixation Time}

The average fixation time was derived by dividing the total length of fixations by the total number of fixations. This would measure the effectiveness of a representation for a participant group in terms of the quick gathering of information necessary for selecting a directory that best matches the query. Fig. (6c) demonstrates that there were main effects of the groups $(F(1,37)=20.56, p<.01)$ and the representations $(F(2,74)=50.54, \quad p<.01)$, and there was interaction between the two factors $(F(2,74)=12.20, p<.01)$. However, in the labeled-pictogram representation, there was no significant difference between the groups. Both groups performed equally well. 


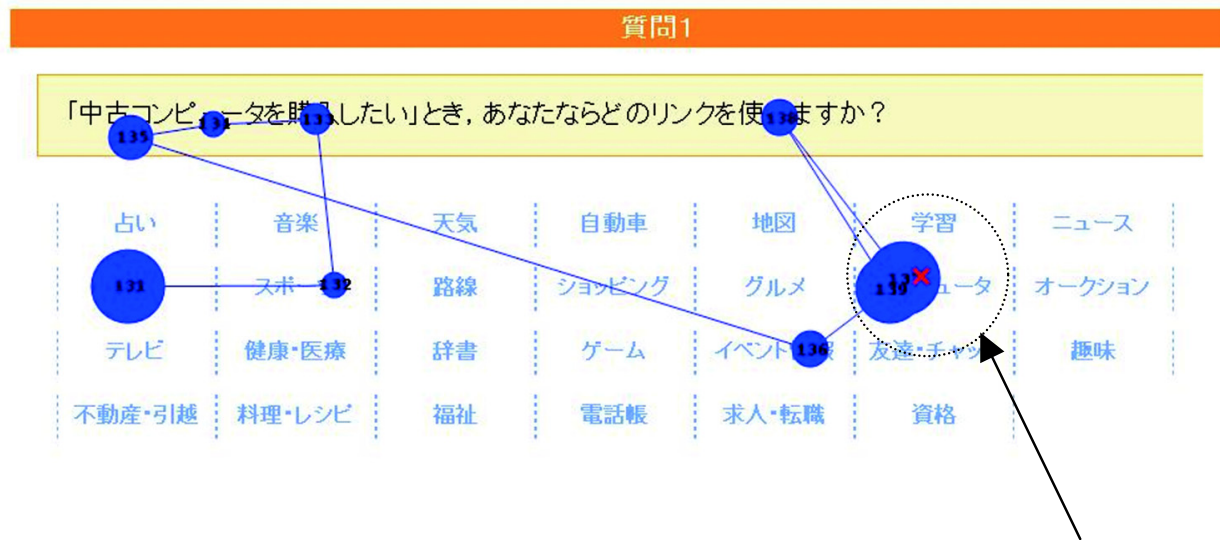

Target: COMPUTER

(a) Text Representation

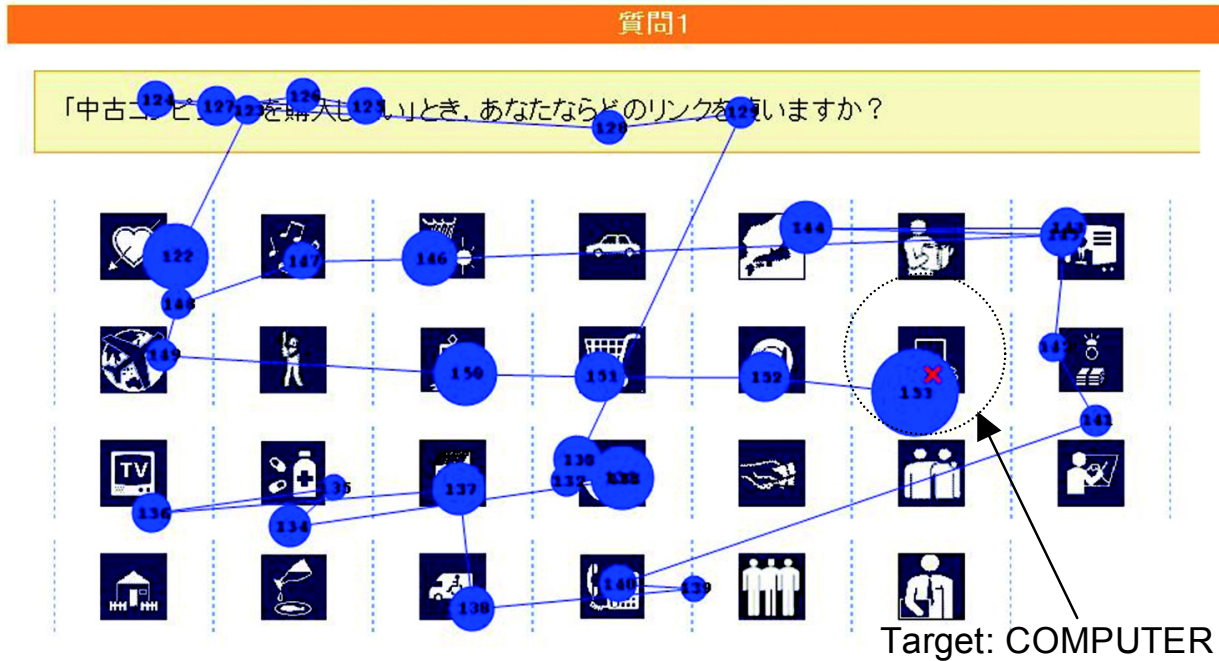

(b) Unlabeled-Pictogram Representation

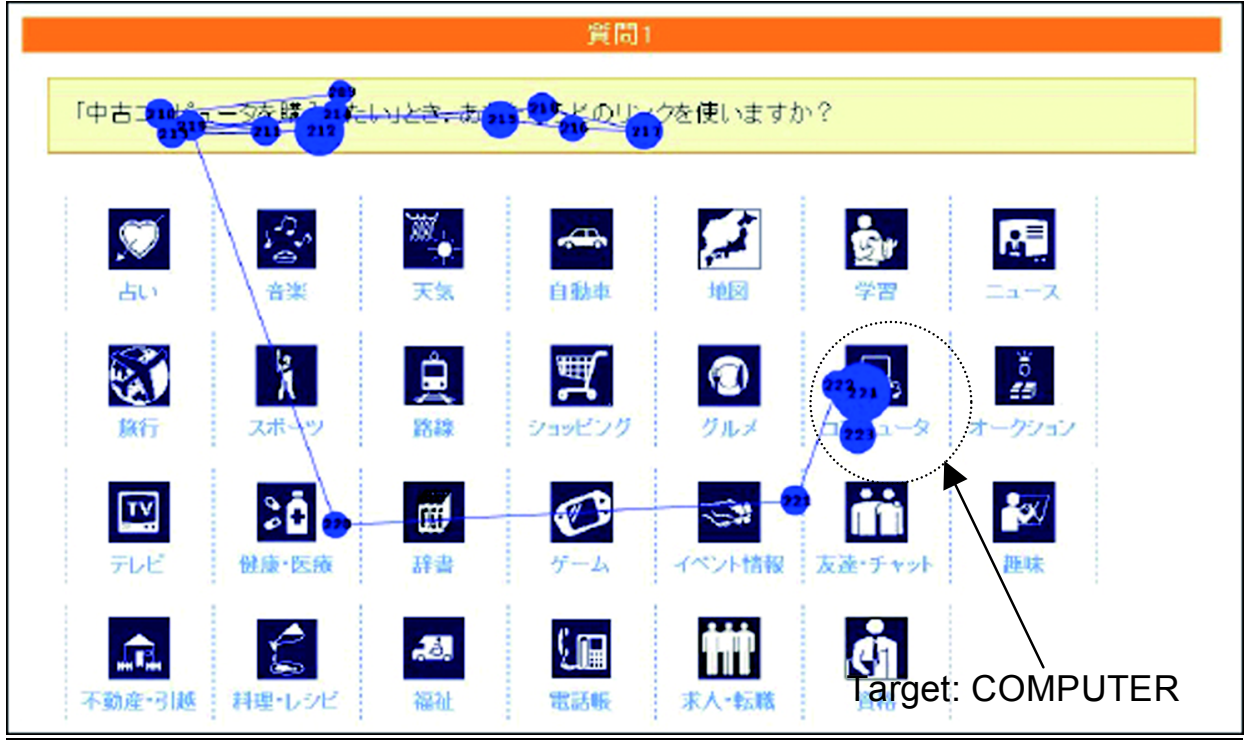

(c) Labeled-Pictogram Representation

Fig. (5). Screen shots of the gaze plots from the deaf participants. 


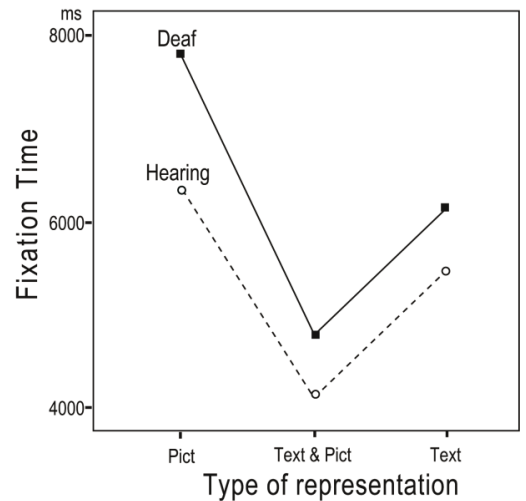

(a) Fixation Time

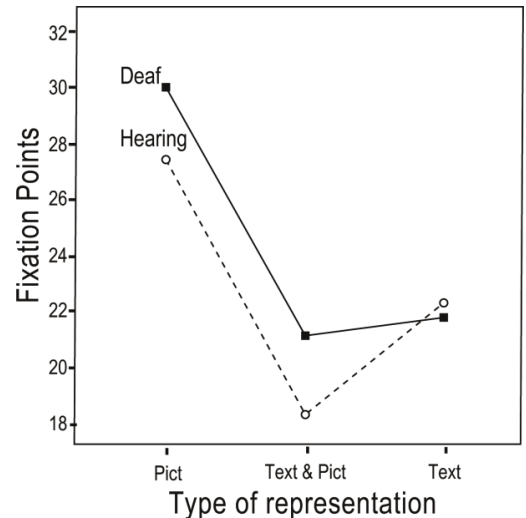

(b) Fixation Points

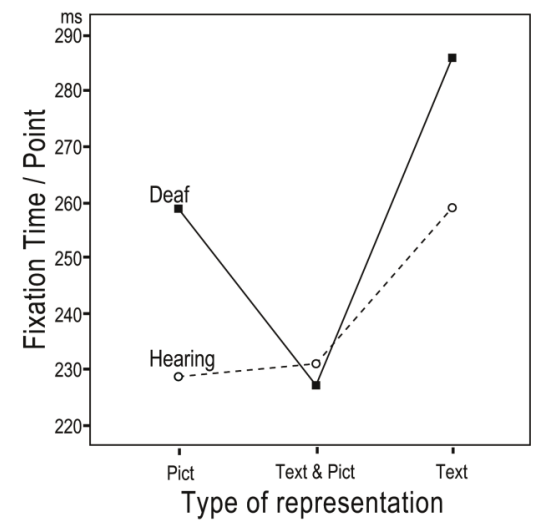

(c) Fixation Time/ Points

Fig. (6). Results of the eye-tracking experiment.

\section{DISCUSSION}

In the following subsections, we summarize the experimental results from the viewpoint of media literacy, i.e., which representation best matches, for example, to persons with superior cognitive skill in processing image representations, i.e., deaf persons. And then, we discuss its implications to designing interfaces for people with disabilities, which is usually carried out by resorting to appropriate guidelines.

\section{Comparison of the Representations}

Unlabeled-pictogram was worse than Labeled-pictogram and Text in terms of speed and variability for both the hearing and the deaf groups. Labeled-pictogram was not significantly different between the hearing and the deaf groups in terms of speed, variability, and eye fixations. Text showed significant difference between the hearing and the deaf groups in terms of eye fixations.

The first part of the analysis on task completion time and the degree of consistency of selections showed the superiority of the labeled-pictogram and the text representations over the pictogram representation; the formers showed shorter task completion time and more consistent directory selection for the queries. The second part of the analysis using eye tracking data showed that the labeled-pictogram representation has advantage over the text representation by showing that the former required the least average fixation time and it showed no difference between the deaf group and the hearing group.

As to comparison of the representations, this study showed the following things:

- Unlabeled-pictogram was worse than Labeledpictogram and Text in terms of speed and variability for both the hearing and the deaf groups.

- Labeled-pictogram was not significantly different between the hearing and the deaf groups in terms of speed, variability, and eye fixations.

- Text showed significant difference between the hearing and the deaf groups in terms of eye fixations.

- Labeled-pictogram and Text were not significantly different for the deaf in terms of eye fixations.
These lead to the conclusion that Labeled-pictogram representation is the best for both the hearing and the deaf groups. Labeled-pictogram representation has advantage over text representation. Participants gathered information necessary to select a directory most efficiently when the directories were represented by labeled-pictograms. Pictogram is useful when quickly recognizing what is represented with limited preciseness and text can add information to make the meaning of the pictogram clearer.

We conjecture that this is because pictogram is useful when quickly recognizing what is represented with limited preciseness and text can add information to make the meaning of the pictogram clearer.

\section{Link Representations and Accessibility Guideline}

Designers of Web-based materials should refer to Web design guidelines from W3C's WAI and the US Government Section 508. The guidelines are presented under "Principles" accompanied by an explanation as to who benefits from them. The primary focus of Web materials and computerbased support for the deaf people is the provision of computer-generated images of sign language and real-time text annotation. For example, the principle 1 of W3C's Web Content Accessibility Guidelines suggests that providing alternatives to audio information is the key to Web accessibility for the deaf. The US Government Section 508 recommends attaching synchronized captions to audio, video, and multimedia material for deaf users. Almost all currently used guidelines involve the substitution of audio information as the only aspect of Web-based interaction. Our previous studies [3] demonstrated that the deaf's style of accessing text information differs from that of hearing persons. Thus, the current use of guidelines for the deaf may be seriously limited.

The results of the current study supplement what is implied by W3C's Principle 3: "content and controls must be understandable." This principle is important because Webbased tasks are performed interactively, requiring comprehension of information provided on the computer screen. This paper focused specifically on three alternative representational formats for navigation links of the Web page which correspond to "control" in the description of W3C's Principle 3. This paper showed that the deaf would gain most benefit if the links are represented as labeledpictograms in a number of performance measures. 


\section{CONCLUSION}

This study showed that labeled-pictogram representation has advantage over text representation. It required least fixation times and least number of fixations. This implies that the deaf and hearing participants gathered information necessary to select a directory most efficiently when the directories were represented by a combination of text and pictogram. We conjecture that this is because pictogram is useful when quickly recognizing what is represented with limited preciseness and text can add information to make the meaning of the pictogram clearer.

The spectrum of media literacy is wide and affects the degree of utility of hyperlinks used in web sites. We will continue on this direction of research to seek optimal multimedia representations for individual visitor groups with specific multimedia literacy.

\section{REFERENCES}

[1] Kurniawan S. Zaphiris P. Research-Derived Web Design Guidelines For Older People: Proceedings Of The 7th International ACM SIGACCESS Conference on Computers and Accessibility. USA: Baltimore, MD 2005; pp. 9-12
[2] Wilson M, Emmorey K. A "word length effect" for sign language: further evidence for the role of language in structuring working memory. Memory Cognit 1998; 26: 584-90.

[3] Namatame M, Nishioka T, Kitajima M. Designing a Web Page Considering the Interaction Characteristics of the Hard-of-Hearing: Proceedings of the 10th International Conference on Computers Helping People with Special Needs. Austria: Lenz 2006.

[4] Namatame M, Kitajima, M. Improving Web Usability for the HardOf-Hearing: Proceedings of Eye Tracking Research \& Applications Symposium. USA: San Diego, California 2006.

[5] Namatame M, Kitajima M. Differences in Web-Interaction Styles of Hard-Of-Hearing and Hearing Persons: Proceedings of the 11th International Conference on Human-Computer Interaction. USA: Las Vegas, Nevada 2005.

[6] Namatame M, Kitajima M, Nishioka T, Fukamauchi,F. A Preparatory Study for Designing Web-Based Educational Materials for the Hearing-Impaired: Proceedings of the 9th International Conference on Computers Helping People with Special Needs. France: Paris 2004.

[7] Namatame M, Nishizaki Y, Kitajima M. Improving Usability of Web Pages for Hard-Of-Hearing Persons: an Investigation of the Utility of Pictograms: Proceedings of the 12th International Conference on Human-Computer Interaction. China: Beijing 2007.

[8] Gentry MM, Chinn KM, Moulton RD. Effectiveness of multimedia reading materials when used with children who are deaf. Am Annals Deaf 2004; 149: 394-402. 